\title{
Gestão de design sistêmica associada à prototipagem de serviço para a tomada de decisão na aplicação de estratégias instrucionais em diferentes contextos educacionais: ludificação e gamificação
}

\author{
Alais Souza Ferreira'; \\ Luiz Fernando Gonçalves de Figueiredo
}

resumo:

Este artigo objetiva descrever as relações existentes entre a gestão de design sistêmica (GDS), a prototipagem de serviço e as estratégias instrucionais (ludificação e gamificação), direcionadas ao contexto de ensino-aprendizagem e/ou treinamentos em diferentes organizações. Para isso, são mencionados três casos educacionais que aplicam essas estratégias instrucionais. Esta pesquisa é de natureza teórica, abordagem qualitativa, objetivos exploratórios. Os dados foram coletados pela pesquisa bibliográfica, incluindo três revisões de literatura realizadas de 2016 a 2018. As pesquisas encontradas permitiram compreender as relações entre essas áreas, resultando em um diagrama sistêmico. A GDS auxilia na prática de gestão, a identificar e colocar em prática a estratégia instrucional adequada para determinado contexto. A prototipagem de serviço propicia o nível tático na visualização dos processos, seja no nível estratégico ou operacional. As estratégias instrucionais contribuem para a instrução da equipe e do usuário, e podem ser aplicadas em todos os níveis de GDS.

palavras-chave:

Gestão de design sistêmica; prototipagem de serviço; estratégias instrucionais; ludificação; gamificação

\footnotetext{
${ }^{1}$ Essa pesquisa foi realizada com apoio da Coordenação de Aperfeiçoamento de Pessoal de Nível Superior Brasil (CAPES) - Código de Financiamento 001.
} 


\section{Introdução}

A pesquisa está voltada para a área de gestão de design sistêmica - baseada na produção, serviços e usuários das comunidades criativas locais, organizações sociais ou educacionais envolvidas nos projetos do Núcleo de Abordagem Sistêmica do Design (NASDesign), pois se notou que a gestão e os serviços desses projetos podem ser aperfeiçoados (BEST, 2012; BERTALANFFY, 2014; CAPRA, 2010; MORIN, 2005; MOZOTA, 2011; SILVA, FIGUEIREDO, 2015). Dentre esses serviços estão o treinamento e o ensino-aprendizagem. Por isso, vislumbrou-se a possibilidade de inserir a ludificação e a gamificação como estratégias instrucionais no nível estratégico de gestão de design sistêmica, para serem utilizadas nos níveis tático e operacional dessa área (ALVES, 2015; DALLAGNOL, 2016; FILATRO, 2008; RAMOS, 1978).

Com isso, questiona-se: como a gestão de design sistêmica associada a prototipagem de serviços está relacionada com as estratégias instrucionais de ludificação e de gamificação para serem aplicadas em contextos de ensino-aprendizagem e/ou treinamentos em diferentes tipos de organizações? Dessa forma, o objetivo deste artigo é descrever as relações existentes entre a gestão de design sistêmica, a prototipagem de serviço e as estratégias instrucionais (ludificação e gamificação) direcionadas ao contexto de ensino-aprendizagem e/ou treinamentos em diferentes organizações. Para isso, são mencionados alguns casos que já aplicam essas estratégias instrucionais.

Esta pesquisa é de natureza teórica, abordagem qualitativa, objetivos exploratórios. Os dados foram coletados por meio da pesquisa bibliográfica, incluindo duas revisões tradicionais da literatura e uma revisão sistemática da literatura (GIL, 2014). Este artigo apresenta, primeiramente, a introdução mencionando o contexto, pergunta, objetivos e caracterização da pesquisa. Na sequência, expõe o conceito de gestão de design sistêmica, de prototipagem de serviços, e das estratégias instrucionais mencionando a ludificação e a gamificação. Em seguida, descreve os procedimentos metodológicos utilizados. Após, relata os resultados encontrados e a discussão dos mesmos. Por fim, menciona as principais considerações finais encontradas.

\section{Gestão de design sistêmica}

$\mathrm{Na}$ contemporaneidade, o reconhecimento da ocorrência de diversidades geográficas, socioculturais e econômicas no Brasil são fatores que têm evidenciado o design, posicionando-o como o responsável pelo desenvolvimento de projetos de produtos, serviços, ambientes e experiências, considerando toda a complexidade e os aspectos peculiares às organizações (educacionais, sociais ou comerciais). $\mathrm{O}$ design envolve pesquisas, processos e práticas centradas nas pessoas, e sua gestão deve ser compreendida como um processo sistemático, que define, planeja, desenvolve, implementa e controla ações de design (AROS; FIGUEIREDO, 2015).

A gestão de design está relacionada à sua inserção na organização ao comunicar a relevância dessa área para as metas de longo prazo e a importância da coordenação dos recursos de design em todos os níveis de gestão (Figura 1), com vistas a atingir os objetivos organizacionais (MOZOTA, 2011; BEST, 2012). A gestão de design abrange os processos, as decisões de negócios e as estratégias que proporcionam a inovação e a criação de produtos, serviços, comunicações e ambientes efetivamente projetados de modo a aprimorar a qualidade de vida da sociedade e propiciar o sucesso organizacional. Assim, a gestão de design vincula diferentes áreas, como design, inovação, tecnologia, gestão e todos os atores envolvidos no processo para fornecer vantagem competitiva nos fatores econômicos, socioculturais e ambientais.

Cada um dos níveis da Figura 1 possui os próprios objetivos, estratégias, metas e medidas de desempenho, definidos de modo independente. Com base em Best (2012) e Mozota (2011), esses níveis de tomada de decisão serão denominados nesta pesquisa como:

- Estratégico - é responsável por determinar a direção estratégica da organização. Por isso, é nesse estágio que se define o escopo e a direção geral que precisam estar alinhadas à visão e à missão da organização. Assim, cada estratégia definida nesse nível representa uma escolha no escopo do design e nas metas organizacionais dos níveis tático e operacional;

- Tático - é a criação de uma função de design na organização e é composto pela administração dos relacionamentos, dos processos, das habilidades para o design, das core competencies e dos treinamentos. Esse estabelece as metas e os objetivos de cada uma das unidades de negócios (linha de produtos ou serviços) ou unidades funcionais (finanças, design, recursos humanos). É nesse nível que o design irá criar valor nos departamentos de apoio da organização, como na gestão de inovação e de tecnologia; 
- Operacional - é o primeiro passo para a integração do design e determina as ações, a execução e as operações diárias, bem como a entrega dos produtos ou serviços. Esse nível envolve o produto, projeto, equipes de clientes ou regiões geográficas, tendo como propósito apoiar igualmente as estratégias organizacionais (nível estratégico) e de negócios (nível tático).

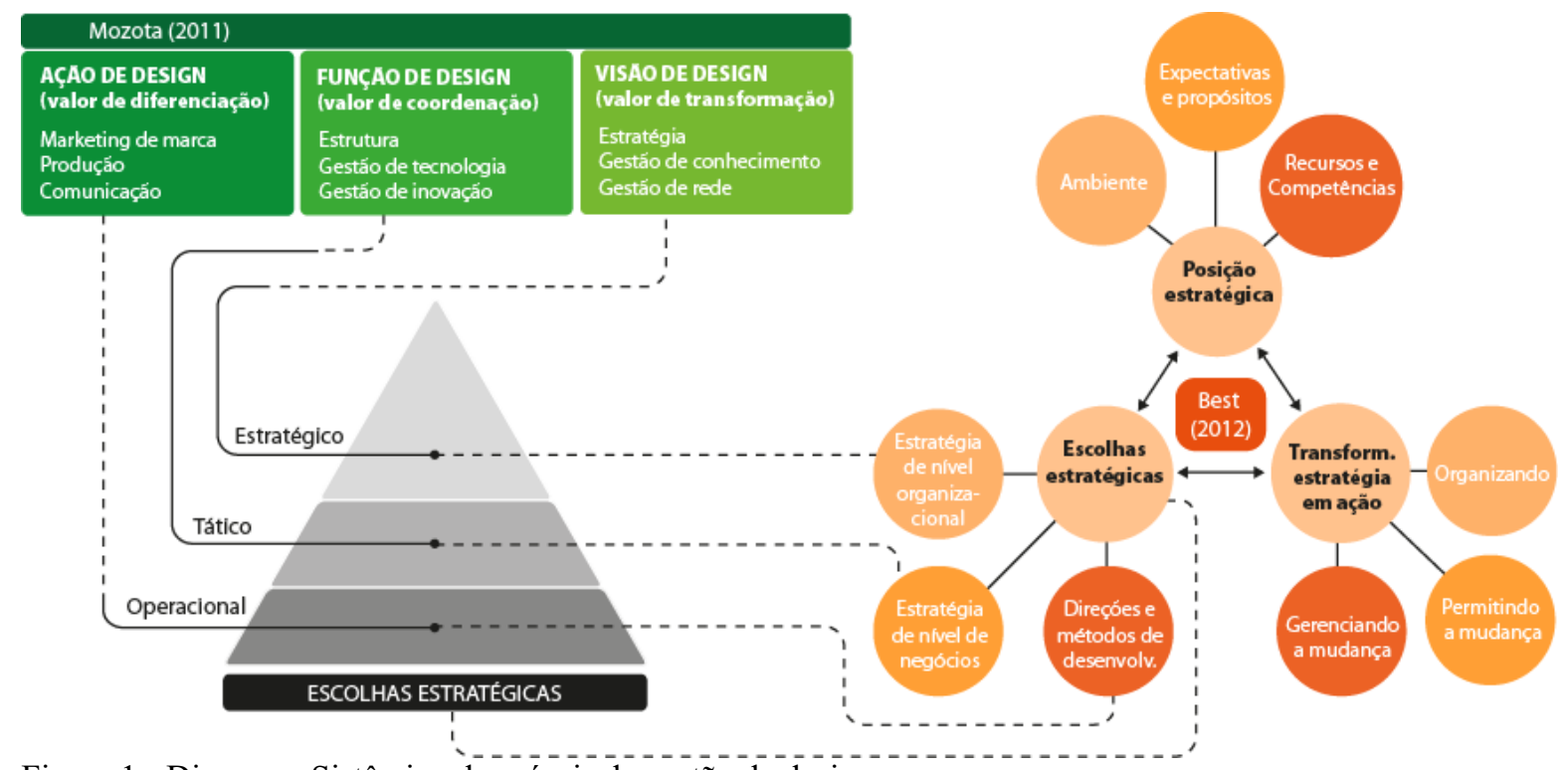

Figura 1 - Diagrama Sistêmico dos níveis de gestão de design.

Fonte: Adaptado de Mozota (2011, p. 310) e Best (2012, p. 79).

Dessa forma, esses níveis orientam os gerentes e os designers a determinarem como diferentes departamentos ou unidades da organização irão desenvolver seus objetivos e estratégias, e como irão escolher agir ao implementar o propósito e as metas gerais (BEST, 2012). Diante dessa percepção, para produzir resultados significativos no planejamento estratégico é fundamental planejar, coordenar, adaptar e revisar as atividades de modo pertinente a cada nível.

As tecnologias e as inovações tecnológicas emergentes "estão impactando a estrutura das organizações em todos os níveis e mudando a maneira como interagem, viabilizam e comportam novas relações, públicos, processos, práticas e formas de engajamento" (BEST, 2012, p. 15). Isso acaba proporcionando inúmeras oportunidades para o design, para a entrega e para a gestão de sistemas interconectados compostos por pessoas, produtos, serviços e experiências.

A gestão de design possui abordagem holística ao integrar a produção de bens, serviços, marketing, recursos humanos, meio ambiente, qualidade de vida, ética e responsabilidade social, com uma visão técnica e estratégica (MOZOTA, 2011). Nesse contexto, a gestão de design é composta pela aplicação das diferentes teorias de gestão (Quadro 1), tanto conceitual quanto prática, para enriquecer os seus métodos.

\begin{tabular}{|l|l|}
\hline \multicolumn{1}{|c|}{ Teorias da Gestão } & \multicolumn{1}{c|}{ Definição } \\
\hline Científica & Gestão de design vista como processo puramente lógico. \\
\hline Comportamental & $\begin{array}{l}\text { Gestão de design como modo de estimular as pessoas a fazer coisas, centrada em } \\
\text { relacionamentos, comportamento interpessoal em grupo e cooperação. }\end{array}$ \\
\hline De decisão & Gestão de design como atividade de tomada de decisão. \\
\hline Sistêmica & $\begin{array}{l}\text { Gestão de design como sistemas de organização com interação aberta com o ambiente e } \\
\text { subsistemas interativos complexos. }\end{array}$ \\
\hline Situacional & Gestão de design depende das circunstâncias. \\
\hline Operacional & $\begin{array}{l}\text { Gestão de design inclui atividades administrativas básicas, como planejamento, } \\
\text { organização, comando, controle e departamentalização. }\end{array}$ \\
\hline
\end{tabular}

Quadro 1 - Teorias de Gestão.

Fonte: Adaptado de Mozota (2011, p. 99).

Na teoria sistêmica está inclusa a abordagem sistêmica que transfere o foco dos objetos para o estudo global dos sistemas. Por isso, envolve todos os seus processos e suas interdependências, uma vez que cada um dos elementos ao serem reunidos para construir uma unidade funcional maior, desenvolvem 
qualidades que não se encontram em seus componentes isolados (BERTALANFFY, 2014). Assim, ao invés de essências e de substâncias, a organização; ao invés das unidades simples e elementares, as unidades complexas; em vez dos agregados formando corpos, os sistemas (MORIN, 2005). Essa visão é considerada holística por conceber o mundo como um todo integrado. Segundo Capra (2010), a ênfase nas partes é chamada de mecanicista ou reducionista; e a ênfase no todo, de holística, organísmica ou sistêmica.

A abordagem de design busca a produção de coerência e tem como critério de sucesso a satisfação da sociedade (BONSIEPE, 2011). Dessa forma, seus resultados podem caracterizar-se como uma inovação sociocultural, que é a percepção sistêmica que qualifica e estimula a atuação do design na contemporaneidade (KRUCKEN, 2009). O principal desafio dessa abordagem é desenvolver soluções à questões de alta complexidade, a fim de mudar a forma de pensar nos projetos mediante uma visão mais ampla, envolvendo produtos, serviços e comunicação, de forma conjunta e sustentável.

A abordagem sistêmica, que está presente no processo de gestão de design, mostra como deve acontecer o trabalho dentro do processo de criação do objeto, seja um produto ou serviço, para que o mesmo seja aperfeiçoado, entendendo que cada parte possui sua importância e deve estar presente dentro de um sistema maior. Nesse contexto, ressalta-se que o design de sistema é fundamental para o sucesso da experiência do usuário com o produto ou serviço (BEST, 2012).

A abordagem sistêmica na gestão de design consiste em utilizar sistemas para compreender a teoria e desenvolver a prática. Por isso: "a busca das inter-relações existentes entre as partes, componentes ou entidades é o foco principal das aplicações teóricas numa abordagem sistêmica, e na gestão de design, este será o foco inicial para entender o funcionamento da gestão de design sob o ponto de vista sistêmico". (SILVA; FIGUEIREDO, 2015, p. 6). A abordagem sistêmica na gestão de design transfere o foco das funções correspondentes a cada nível de atuação, para as interações presentes no sistema formado pela gestão de design. Isso torna necessário visualizar quais são os principais atores (componentes/entidades) da gestão de design, já que é entre eles que acontecem as interações (SILVA, FIGUEIREDO, 2015). Os autores constataram por meio da observação em campo que o tipo e a qualidade dessas interações do sistema dependem da dinâmica de funcionamento e da cultura da organização. Assim, as interações entre as partes são inúmeras, possuindo muitas entradas, saídas e trocas de informação. Por esse motivo, a organização precisa ter métodos e ferramentas para coordenar essas interações, de maneira a manter o sistema em equilíbrio (homeostase).

Portanto, a gestão de design sistêmica promove "o gerenciamento bem-sucedido de pessoas, projetos, processos e procedimentos que estão por trás da criação dos produtos, serviços, ambientes e experiências que fazem parte de nossa vida diária" (BEST, 2012, p. 8). Isso também envolve a gestão das relações entre diferentes áreas (design, gestão, marketing e financeiro) e atores (clientes, designers, equipes de projeto, entre outros). Assim, a implementação do planejamento de design na organização é realizada com o intuito de ajudá-la a alcançar os objetivos, a melhorar a qualidade dos produtos e serviços e a qualificar as equipes.

O estudo desenvolvido por Silva e Figueiredo (2015, p. 12) mostrou que a gestão de design sistêmica permite: "visualizar o todo em que se está inserido, o que pode ajudar no processo de desenvolvimento de estratégias, melhoria em processos e desenvolvimento de projetos com grande abrangência, bem como ser aplicada no contexto diário, como forma de organizar e planejar", as atividades do dia a dia dos projetos. Logo, a gestão de design sistêmica contribui em todos os níveis da tomada de decisão: no nível estratégico, por ajudar os líderes a visualizarem a organização como um todo, percebendo os pontos fortes e fracos em que as interações estão frágeis e suscetíveis a gerar problemas; e nos níveis tático e operacional, por auxiliar na organização de processos, ajudar o gestor de design e o designer a entenderem o processo do qual fazem parte, e por permitir a sistematização de suas tarefas como forma de organização e eficácia em sua atuação. $\mathrm{O}$ próximo item aborda a prototipagem de serviço e, na sequência, as estratégias instrucionais.

\section{Prototipagem de serviços}

Inserido nesse contexto, encontra-se o design de serviço, que ajuda a inovar, criar novos, ou aperfeiçoar serviços, tornando-os mais úteis, usáveis e desejáveis para os clientes, bem como eficientes e eficazes para as organizações. É um novo campo holístico, multidisciplinar e integrativo, por conectar as áreas de marketing, design, gestão de empresa e pesquisa de cliente (MORITZ, 2005). O design de serviço: "aplica métodos centrados no usuário para inovar e dar forma aos serviços organizados em torno das 
necessidades e desejos das pessoas, levando em consideração as restrições e as possibilidades dos provedores de serviços e ambientes" (BOHREN et al., 2015, p. 5, tradução nossa).

O serviço é qualquer ato ou desempenho que uma parte possa oferecer a outra, que seja essencialmente intangível e não resulte na propriedade de nada (KOTLER, 2005). No entanto, a execução de um serviço pode ou não estar ligada a um produto físico. É complicado testar e avaliar as características de um serviço, já que o mesmo não possui uma forma física, principalmente serviços baseados em complexas interações sociais. Por isso, as ideias complexas necessitam de protótipos que possam ser testados em campo para verificar como sobrevivem e se adaptam, o que seria a prototipagem de serviço (BROWN, 2010). O autor relata que prototipar significa dar forma a uma ideia, permitindo aprender, avaliar e aperfeiçoar essa ideia em relação a outras. A jornada do usuário é um exemplo de estrutura simples, que mapeia os estágios pelos quais um cliente imaginário passa do início ao final de uma experiência no desenvolvimento de novos serviços.

A palavra protótipo deriva do grego prototypos que é a junção dos termos proto (primeiro) e typos (impressão) (BLOMKVIST, 2011). Essa palavra é utilizada em diferentes contextos e disciplinas, inclusive no design. Geralmente, a prototipagem beneficia o processo de design e a produção. Os protótipos de serviço permitem visualizar os processos e as interações, ao invés de objetos tangíveis. Por isso, é necessário selecionar métodos de prototipagem adequados para apoiar a ideação colaborativa. Os protótipos de serviço são úteis na ideação e podem auxiliar na superação dos desafios apresentados pela imaterialidade e pela interatividade dos serviços, tornando possível que os designers mapeiem o processo completo (RAU; ZBIEK; JONAS, 2017).

Zimmer Santos e Hoffmann (2016) informam que por meio do protótipo é possível estabelecer o escopo do serviço a ser implantado, para o qual todo o projeto será elaborado. Além disso, quando relacionado com o serviço, produzir um protótipo torna-se "uma maneira informativa para designers explorarem quais seriam as experiências para uma determinada pessoa, num contexto específico, por meio da improvisação dramática" (CASTRO, 2014, p. 77). Logo, o protótipo proporciona uma experiência realista para os usuários, exigindo certo grau de resolução e funcionalidade condizente com o contexto cotidiano e que esteja plenamente integrado à vida das pessoas (CASTRO, 2014). Por isso, a prototipagem da experiência é aquela: "aplicada para demonstrar o contexto, identificar problemas e oportunidades de design. [...] o objetivo da prototipagem é alcançar uma simulação de alta fidelidade de uma experiência existente, que não pode ser experimentada diretamente, porque é insegura, indisponível, custa caro, etc." (CASTRO, 2014, p. 76).

Assim, a prototipagem e os protótipos são relevantes no desenvolvimento de novos produtos e, especialmente, dos serviços, que são intangíveis. Porém, desde a conceituação de prototipagem, existe uma preocupação em relação à fidelidade. $\mathrm{Na}$ área de serviços, o termo fidelidade do protótipo está relacionado com o nível de proximidade da experiência projetada, ou da jornada do usuário, com a situação real, pois é nela que estão presentes as funcionalidades, os aspectos estéticos, a usabilidade, entre outros (HOSS, 2014). O autor compila em um infográfico as taxonomias de protótipos (Figura 2), que classifica o estágio do protótipo e as diferentes posições que um protótipo pode assumir (fidelidade, viabilidade e impacto de cocriação, custo de mudança), nas fases do processo de design.

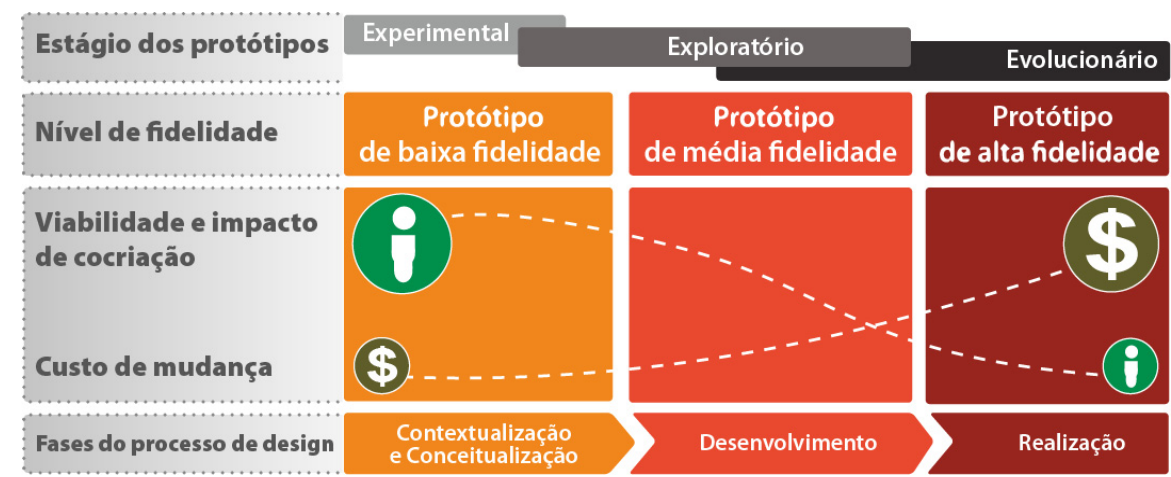

Figura 2 - Taxonomia de protótipos.

Fonte: Hoss (2014, tradução nossa).

Logo, a prototipagem, quando relacionada aos serviços, busca simular as experiências considerando o provedor do serviço, os artefatos envolvidos, o usuário e suas expectativas, e o ambiente 
(HOSS, 2014). Desse modo, os protótipos tornam visíveis as ideias de serviços para os atores envolvidos, fornecendo "um ponto de referência comum, que permite que os interessados em colaborar e avaliar forneçam sugestões para aperfeiçoar o projeto (artefatos ou serviços)" (HOSS, 2014, p. 45, tradução nossa). Dentre esses serviços que precisam ser prototipados estão os serviços de ensinoaprendizagem e treinamento de diferentes organizações educacionais, sociais ou corporativas. Para a prototipação desses serviços é necessário estabelecer estratégias instrucionais.

\section{Estratégias instrucionais: ludificação e gamificação}

As estratégias instrucionais estão relacionadas ao design instrucional. Esse é definido "como o processo (conjunto de atividades) de identificar um problema (uma necessidade) de aprendizagem e desenhar, implementar e avaliar uma solução para esse problema" (FILATRO, 2008, p. 4). Essa definição considera que o design é o resultado de um processo ou atividade, enquanto a instrução é a atividade de ensino que utiliza a comunicação de modo que facilite a aprendizagem. Assim, devido à integração de três ciências (humanas, da informação e da administração) relacionadas à aprendizagem e ao comportamento humano, torna-se possível compreender como a informação pode ser combinada, processada e apresentada de modo criativo e específico, considerando o contexto histórico, social e organizacional mais amplo (FILATRO, 2008).

A estratégia instrucional, segundo Ramos (1978), é um termo amplo e genérico que se refere aos modos usados para implementar a instrução, permitir que os propósitos sejam atingidos e estabelecer o como fazer para que o aluno aprenda, ou seja: Que procedimentos utilizar? Que meios empregar? E que eventos efetivar? Cada componente das estratégias instrucionais possui aspectos específicos que são utilizados para ensinar (Figura 3).

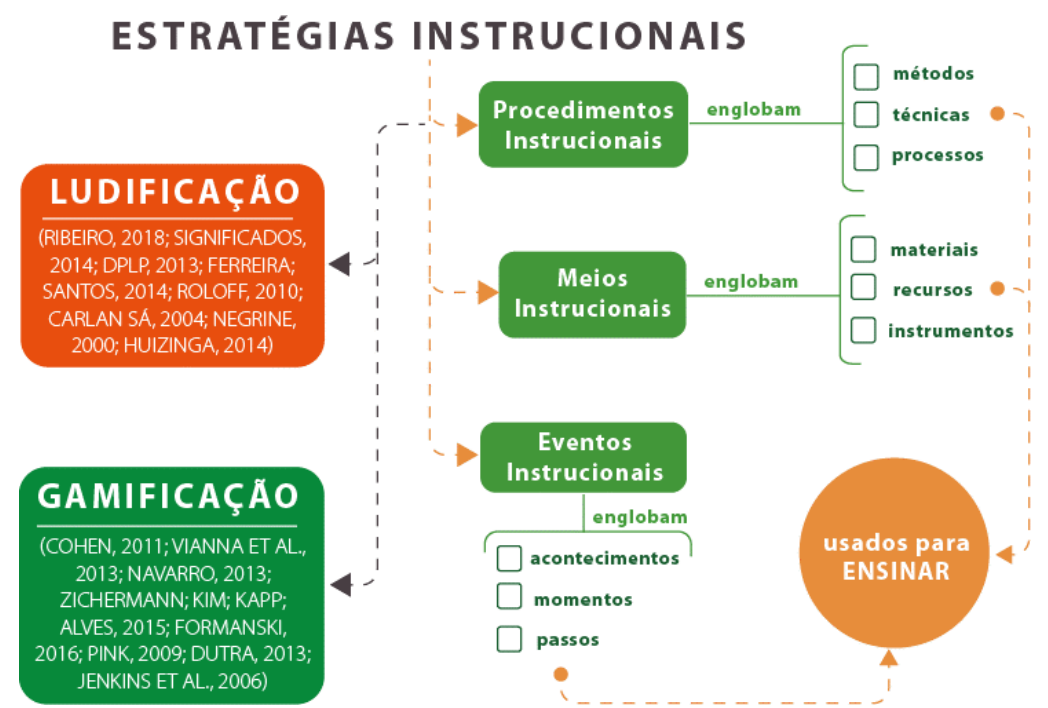

Figura 3 - Componentes das estratégias instrucionais.

Fonte: Adaptado de Ramos (1978, p. 19-20).

A pesquisa compreende que as estratégias instrucionais são ações planejadas para o desenvolvimento do processo de instrução ou aprendizagem, que permitem o alcance dos objetivos estabelecidos (RAMOS, 1978). Nessa abordagem, o usuário deve estar no centro do processo, pois as pessoas não "percebem o mundo da mesma forma, comunicam-se da mesma maneira, aprendem do mesmo modo ou jogam um determinado jogo de um único jeito" (ALVES, 2015, p. 75). As estratégias são compostas por três itens utilizados para ensinar: os procedimentos instrucionais (métodos, técnicas, processos); os meios instrucionais (materiais, recursos, instrumentos); e os eventos instrucionais (acontecimentos, momentos, passos). A ludificação e a gamificação podem estar inclusas nos procedimentos instrucionais.

Tanto a ludificação quanto a gamificação são vistas nesta pesquisa como estratégias instrucionais, que são formas interativas e divertidas, para alcançarem um objetivo específico e mensurável. Por esse motivo, o desenvolvimento de uma solução de aprendizagem ludificada ou gamificada, seja um treinamento ou uma aula, deve começar pela definição dos objetivos e das 
estratégias. Isso contribuiu para a medição do resultado e ajuda a seguir um fluxo completo, até que a aprendizagem seja transferida para o local de trabalho e gere o impacto desejado (ALVES, 2015). A autora ainda menciona que a aprendizagem nas organizações está relacionada a vários tipos de conhecimentos (como a resolução de problemas ou os processos de tomada de decisão). Por isso, cada um desses conhecimentos exige a escolha de uma estratégia instrucional diferente para ensiná-los. Isso também se aplica na escolha das técnicas de design, que serão utilizadas na ludificação ou na gamificação, pois uma determinada técnica pode não ser aplicável em tudo o que é necessário ensinar.

Portanto, quando uma demanda relacionada a esse serviço chegar, é necessário primeiramente compreender qual é o problema e a partir daí verificar a melhor estratégia instrucional. No entanto, os profissionais envolvidos com a aprendizagem precisam exercitar a empatia antes mesmo de entender o problema, uma vez que pode ocorrer da solicitação pelo serviço já chegar com uma estratégia instrucional definida. Nesse caso, não se pode culpar o solicitante, é necessário descobrir o problema e, se essa não for a melhor estratégia instrucional, é fundamental mostrar as razões pelas quais outra estratégia irá adequar-se melhor ao objetivo que se quer atingir (ALVES, 2015).

Por fim, constata-se que para decidir entre a ludificação e a gamificação, como a estratégia instrucional mais adequada é necessário passar pelo processo de um design instrucional bem detalhado. Na visão de Alves (2015, p. 147), o sucesso depende "da utilização de um sistema de design instrucional completo, que seja capaz de, em sua amplitude, abranger desde a identificação dos objetivos e sistemas de avaliação até a análise e documentação dos resultados". Esta pesquisa limita-se a apresentar apenas duas estratégias instrucionais: a ludificação e a gamificação, por entender que essas duas estão direcionadas para os Projetos do NASDesign.

\subsection{Ludificação}

A ludificação é um processo que tem como principal propósito o divertimento (RIBEIRO, 2018). Esse processo permite ao usuário exercer livremente a sua vontade e pode evocar seus sentimentos de despreocupação, de liberdade, de espontaneidade de ação e de alegria (HUIZINGA, 2014). Ele, também, contribui para o desenvolvimento afetivo, motor, mental, intelectual e social do indivíduo, que compreende o mundo por meio da construção e reconstrução da espontaneidade (FERREIRA; SANTOS, 2014). Esse processo pode utilizar atividades lúdicas como jogos (sejam pedagógicos ou não), brincadeiras, atividades criativas, dinâmicas em grupo, cantigas de roda, colagens, entre outros (RIBEIRO, 2018; ROLOFF, 2010; CARLAN SÁ, 2004). Essas atividades lúdicas são ações de entretenimento que dá prazer, que representam o ato de brincar ou jogar e que não se reduzem apenas as atividades infantis (SIGNIFICADOS, 2014). Elas também são despretensiosas, descontraídas e desobrigadas de toda e qualquer intenção ou vontade pertencente a outras pessoas. Por isso, essas atividades são livres de pressões, avaliações e responsabilidades. (NEGRINE, 2000). Além disso, elas podem manter o indivíduo afastado das atividades produtivas (no sentido de possuírem uma intencionalidade final) por certo período (CARLAN SÁ, 2004).

Assim, compreende-se as ações de ludificar como o uso de atividades não estruturadas que ocorrem de modo espontâneo e que são inerentes ao instinto natural dos seres vivos, de se relacionar, se divertir e se preparar para atividades mais complexas que acontecerão no futuro. Essas atividades são fundamentais para o desenvolvimento do indivíduo durante os processos de aprendizagem e investigação das relações com o mundo e com a sociedade. $\mathrm{O}$ ato de brincar ou jogar também pode estar presente nas atividades dos adultos, por ser um processo mental que depende do indivíduo e que é despertado por meio de estímulos durante as ações. Esse ato é relevante para os adultos, conforme o estudo do psicólogo americano e neurocientista Jaak Panksepp, devido o ator de brincar, inicialmente, ativar o tronco cerebral que é onde os mecanismos de sobrevivência são originados - como a respiração, a consciência, o sono e os sonhos. Essa ativação aciona as emoções prazerosas que estão inclusas no ato de brincar. (ALVES, 2015). Portanto, sem essa conexão não existe o ato de brincar ou jogar. Tendo isso em vista, ressalta-se que essa ativação se faz presente na vida adulta e é realizada em diversas circunstâncias e ações, incluindo o trabalho. 


\subsection{Gamificação}

A gamificação vem sendo compreendida como a aplicação de elementos (mecânicas, dinâmicas e técnicas) de jogos em situações pertencentes à realidade do dia a dia profissional, escolar e social do indivíduo. Como exemplos de elementos de jogos, menciona-se o lançamento de desafios, cumprimento de regras, metas claras e bem definidas, efeito surpresa, linearidade dos acontecimentos, conquista por pontos e troféus, estatísticas e gráficos com o acompanhamento da performance, superação de níveis e criação de avatares, entre outros. Cada um desses elementos é apenas uma parte do sistema gamificado e não o todo. Por isso, deve-se estudar o contexto para compreender qual é a combinação ideal para cada caso. (ALVES, 2015; COHEN, 2011; NAVARRO, 2013; VIANNA et al., 2013).

O processo de gamificação pode ser a combinação da diversão (ludificação) com os elementos de jogos. Como estratégia instrucional, visa criar envolvimento entre o indivíduo e determinada situação, com $\mathrm{o}$ intuito de favorecer a mudança de comportamento, aumentando o interesse, o engajamento, a motivação e a eficiência na realização de uma tarefa específica ou na resolução de um problema. (ALVES, 2015; COHEN, 2011; NAVARRO, 2013; VIANNA et al., 2013). A gamificação possui três fatores principais: a motivação (extrínseca e intrínseca), estímulo e habilidade (FORMANSKI, 2016; PINK, 2009; DUTRA, 2013; JENKINS et al., 2006). A seguir, os procedimentos metodológicos são detalhados.

\section{Procedimentos metodológicos}

Este artigo é o resumo de uma pesquisa teórica desenvolvida para fundamentar uma dissertação de mestrado. A pesquisa foi desenvolvida mediante a consulta de sites, periódicos, artigos, dissertações, teses e livros. Para isso, foram realizadas três revisões de literatura com base na definição de Conforto, Amaral e Silva (2011). A primeira, caracterizada como revisão tradicional da literatura, ocorreu de 2016 a 2017, abrangendo as áreas de gamificação, gestão de design, abordagem sistêmica e design de serviço com ênfase em prototipagem. O banco de dados utilizado para essa revisão foi o Google Acadêmico.

A segunda, caracterizada como revisão sistemática da literatura, ocorreu em 2017 abrangendo as mesmas áreas da revisão anterior. Os bancos de dados consultados foram a Scopus, a Scielo, a Science Direct, a Web of Science, a Capes e a Proquest. Os critérios de exclusão foram quanto ao tipo de referência (resumos), acesso (arquivos pagos ou obtidos ilegalmente), escopo (pesquisas fora da área de design), data (pesquisas publicadas antes de 2012) e idioma (pesquisas não escritas em português, inglês ou espanhol). O resultado bibliométrico está publicado no artigo de Figueiredo, Ferreira e Conti (2018).

A terceira, caracterizada como revisão tradicional da literatura, ocorreu em 2018 no Google Acadêmico, sem filtros, abrangendo somente a área de ludificação. $\mathrm{O}$ intuito foi identificar pesquisas que subsidiassem a diferença entre ludificação e gamificação, e para entender o conceito de ludificação. Nessa busca não foram encontradas publicações relevantes. Por isso, a pesquisa continuou na internet e junto a profissionais da área, buscando livros que ajudassem a compreender as diferenças como o "Homo Ludens" de Huizinga (2014). Buscaram-se também definições em diversos dicionários que ajudassem a compreender o conceito de ludificação. Além disso, realizou-se uma consulta nas pesquisas que vieram nas duas primeiras revisões da literatura e encontrou-se um artigo dos autores Ferreira e Santos (2014). No entanto, o propósito deste artigo é mesclar essas três revisões de literatura, analisar essas pesquisas e descrever a interpretação encontrada referente a relação existente entre essas áreas.

\section{Resultados e discussões}

O design é um agente de mudança que pode se apropriar de estratégias instrucionais (a ludificação e a gamificação) que contribuem para evidenciar as competências, as habilidades e as capacidades de cada ator. Isso ocorre porque essas estratégias instrucionais visam à mudança de comportamento. E, por isso, auxiliam o gestor na coordenação sistêmica de todos os envolvidos no processo de gestão de design. Além disso, podem auxiliar o gestor a equilibrar tanto as situações externas (como a compreensão do ambiente competitivo e das necessidades dos consumidores) como as internas (objetivos organizacionais, gerenciamento de projetos, critérios de custo, etc.). A abordagem sistêmica possui visão holística (CAPRA, 2010) que, por sua vez, está implícita na gestão de design (MOZOTA, 2011), constituindo o que entendemos por gestão de design sistêmica. Enquanto a prototipagem de 
serviço e as estratégias instrucionais enquadram-se nas escolhas estratégicas inclusa na direção estratégica de gestão de design. Evidenciam-se essas relações na Figura 4.

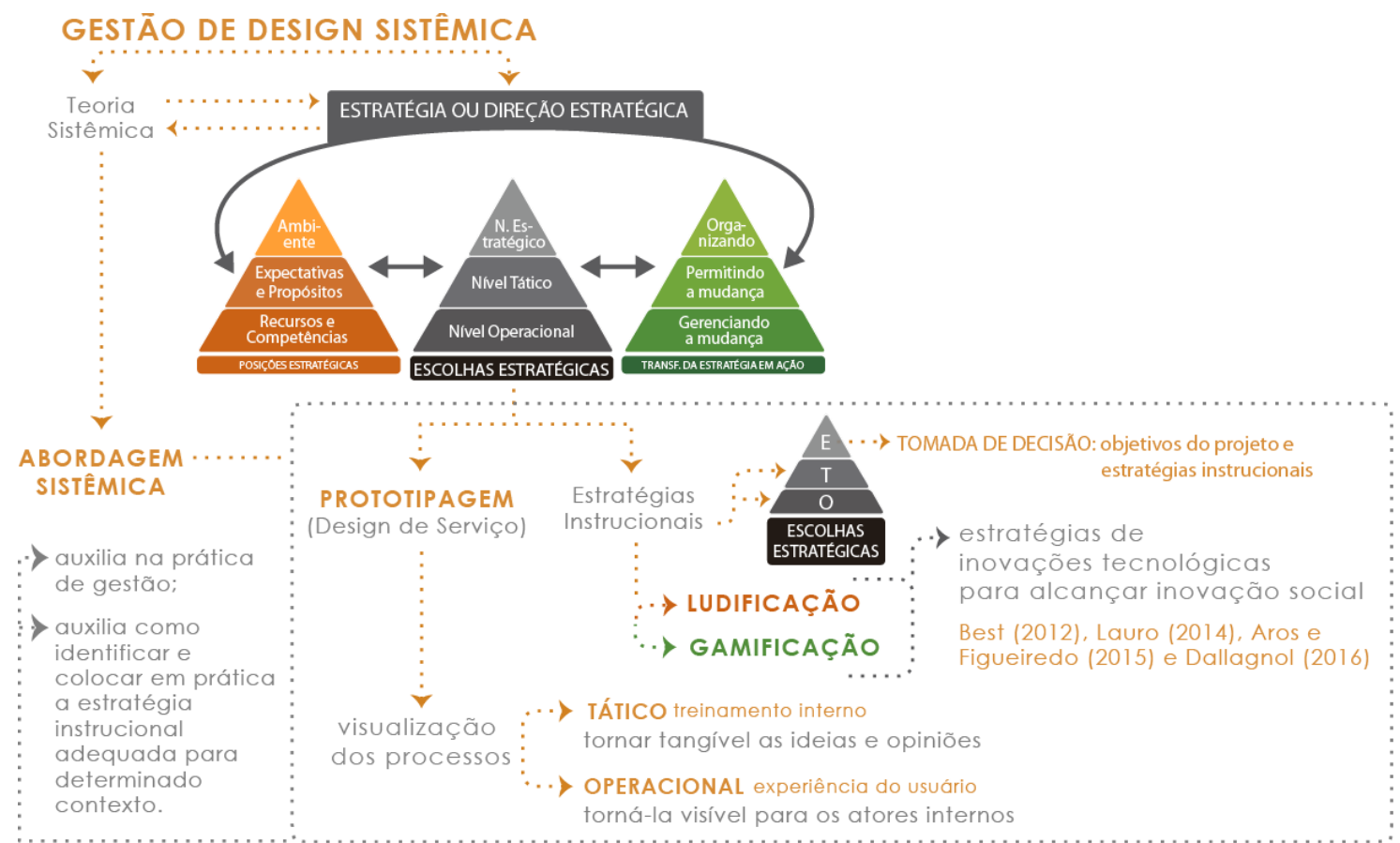

Figura 4 - Diagrama Sistêmico com a síntese das relações entre os conceitos.

Fonte: Elaborada pelos autores.

Para Best (2012) e Mozota (2011), a estratégia de uma organização ou projeto é construída com base nos níveis estratégico, tático e operacional inclusos nas escolhas estratégicas (Figura 4). Cada um desses níveis possuem os próprios objetivos, estratégias, metas e medidas de desempenho, definidas de modo independente. Assim, a decisão das estratégias instrucionais (ludificação e gamificação) está inclusa nas escolhas estratégicas, já que as decisões são tomadas após as posições estratégicas terem sido definidas, o que inclui o estabelecimento dos objetivos que se almeja atingir.

Além disso, a abordagem sistêmica auxiliou na compreensão de todas as áreas que estão envolvidas na pesquisa. Ela também ajudou a entender como que a gestão de design, a prototipagem de serviço, e as estratégias instrucionais (ludificação e gamificação) estão relacionadas, quais são as convergências e as divergências entre essas áreas (Figura 5) (BERTALANFFY, 2014; BEST, 2012; CAPRA, 2010; KRUCKEN, 2009; MORIN, 2005; SILVA; FIGUEIREDO, 2015; LAURO, 2014). Por esse motivo, a abordagem sistêmica está inclusa nesta pesquisa e se faz necessária no processo de gestão de design que está inserido nas organizações educacionais.

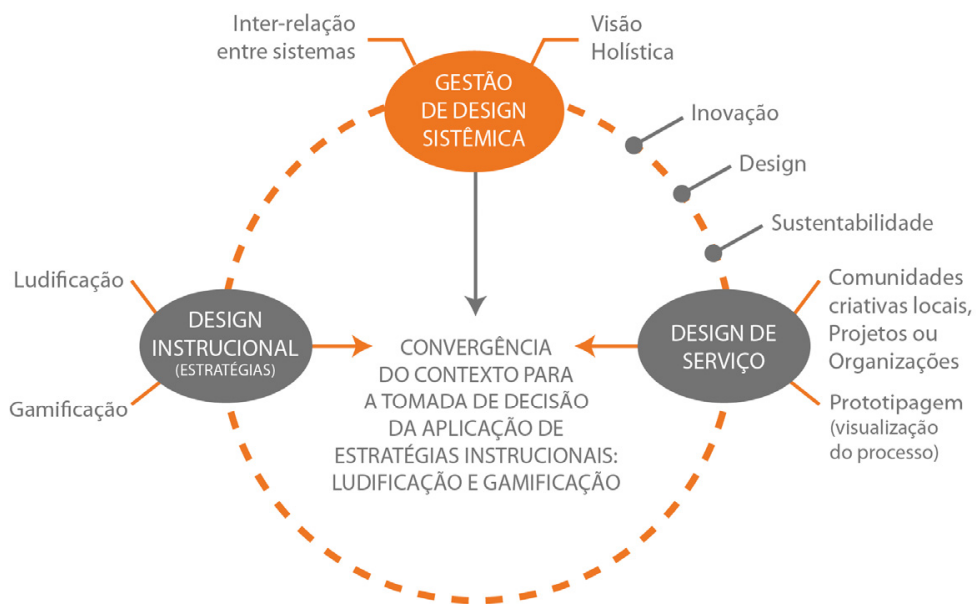

Figura 5 - Relação entre as áreas inclusas na pesquisa.

Fonte: Elaborada pelos autores com base em Ferreira, Figueiredo e Ourives (2017). 
Após o cruzamento das pesquisas, percebeu-se que a convergência encontrada é que todas as áreas inclusas neste artigo (gestão de design sistêmica, design de serviço e design instrucional) mencionam que primeiramente é necessário definir os objetivos e fazer um diagnóstico detalhado do contexto, para que depois sejam definidas todas as estratégias instrucionais ou não. Todos os autores citados anteriormente e as áreas pesquisadas abordam a mesma informação, porque todas essas áreas tiveram origem no design. A junção do design com a gestão deu origem a gestão de design. $\mathrm{O}$ design de serviço inclui a prototipagem de serviço. $\mathrm{O}$ design instrucional abrange as estratégias instrucionais. $\mathrm{O}$ design lúdico deu origem a ludificação. E o design de jogos proporcionou o surgimento da gamificação. Somado a isso, é perceptível que todos os processos e as etapas metodológicas de prototipagem de serviço, design lúdico e design de jogos derivam da metodologia de design.

As divergências encontradas estão relacionadas à ludificação e a gamificação. A ludificação não precisa ter uma estrutura, é livre, podendo ser composta por um jogo, uma brincadeira ou uma dinâmica simples, sem que o usuário tenha a percepção de que está participando de uma aprendizagem ou treinamento. O contexto de aplicação da ludificação é voltado para o divertimento e o entretenimento, mas também podem ser aplicados em situações de não entretenimento. Já a gamificação tem que ser obrigatoriamente estruturada com a aplicação do pensamento de jogos (incluindo as mecânicas, dinâmicas, componentes e estéticas) em situações reais do dia a dia das pessoas, podendo ou não resultar em um jogo. O contexto de aplicação da gamificação está direcionado obrigatoriamente para situações de não entretenimento.

Assim, ao analisar e juntar as pesquisas de Lauro (2014) e Dallagnol (2016), percebe-se que as estratégias instrucionais se inserem nos níveis tático e operacional da gestão de design sistêmica (Figura 4). Já que o nível tático está direcionado ao treinamento dos atores e aos processos internos organizacionais ou projetuais. E o operacional está mais voltado para os usuários ou consumidores finais. Dessa forma, as estratégias instrucionais podem estar tanto no nível de treinamento interno como no nível de experiência do usuário ou do consumidor final.

Assim, o desenvolvimento de uma solução de aprendizagem ludificada ou gamificada deve começar pela definição de objetivos e estratégias para a medição do resultado e seguir o fluxo completo até que a aprendizagem seja transferida para o local de trabalho e possa gerar o impacto esperado na organização (ALVES, 2015). Por isso, o sucesso da ludificação ou da gamificação depende de um bom diagnóstico do contexto e da definição de objetivos.

Logo, as estratégias instrucionais (ludificação e gamificação) podem ser consideradas tecnologias e inovações tecnológicas emergentes. Elas têm impactado a estrutura das organizações em todos os níveis, mudando a forma como interagem, viabilizam e comportam novas relações, públicos, processos, práticas e formas de engajamento. Isso ocorre porque tanto a ludificação quanto a gamificação colaboram na diferenciação e no aperfeiçoamento das experiências, seja na entrega do serviço ou do produto. Portanto, a ludificação e a gamificação podem ser consideradas estratégias de inovações tecnológicas para alcançar a inovação social, baseada na gestão de design sistêmica. (AROS; FIGUEIREDO, 2015; BEST, 2012; DALLAGNOL, 2016; LAURO, 2014).

A partir disso, a prototipagem de serviço auxilia na visualização dos processos, na parte tática, para que as pessoas que estão participando do treinamento ou do processo de gestão interna da organização ou do projeto possam compreender a opinião dos demais e a equipe possa falar a mesma linguagem. Ou seja, todos os participantes possam entender o que cada um está pensando ou falando, por meio de uma representação gráfica tangível, tornando as ideias, as opiniões e o processo mais tangíveis. Na parte operacional, a prototipagem de serviço auxilia na visualização do processo ou da experiência do usuário ou consumidor final, para os atores internos. Assim, esses que estão trabalhando na parte operacional (gestores e designers) conseguem compreender como está sendo a experiência para os usuários e, a partir disso, conseguem identificar as falhas ou os acertos e aperfeiçoar essa experiência, além de conhecer como interferem na experiência do usuário.

As estratégias instrucionais são as maneiras usadas para implementar a instrução e permitir que os objetivos sejam alcançados (RAMOS, 1978). Assim, entende-se que as estratégias instrucionais são ações planejadas para o desenvolvimento do processo de instrução ou aprendizagem, em função dos objetivos a serem alcançados. É importante destacar que nesse tipo de abordagem o usuário deve estar no centro do design. Elas possuem procedimentos instrucionais (métodos, técnicas, processos), meios instrucionais (materiais, recursos, instrumentos) e eventos instrucionais (acontecimentos, momentos, passos), sendo que todos são usados para ensinar. A ludificação e a gamificação enquadram-se nos procedimentos instrucionais. 
Essas estratégias instrucionais auxiliam na gestão de relações entre diferentes áreas e atores, bem como na administração, valorização e promoção das pessoas necessárias na gestão de design sistêmica (BEST, 2012). Além disso, a gamificação e a ludificação podem auxiliar no desenvolvimento das competências que a equipe de design precisa ter, bem como no desenvolvimento das habilidades essenciais para o gestor de design. Dessa forma, a ludificação e a gamificação despertam a motivação e o comprometimento dos envolvidos no processo de gestão de design, auxiliando na transformação da cultura da organização ou do projeto.

Ao relacionar as estratégias instrucionais com os níveis de gestão de design, percebeu-se que a ludificação pode ser utilizada como uma estratégia de gestão de processo interno (tático), como um produto que a empresa oferece (operacional), ou até como uma filosofia, uma missão, uma visão de mundo, que orienta todo o restante (estratégico). Já a gamificação está mais direcionada para o como fazer (tático), em relação à organização do processo, seja para o estratégico ou para o operacional. No entanto, já existem casos na área de educação que utilizam tanto a ludificação quanto a gamificação em todos os níveis de gestão de design como: a Perestroika; a Quest to Learn; e a Vittra Telefonplan School.

Ao pesquisar a Perestroika ${ }^{2}$ (Figura 6), localizada no Brasil, percebeu-se que a missão, a visão e os valores da empresa estão direcionados para a ludificação e para a gamificação. Atualmente, em 2020, os profissionais: possuem o propósito de ajudar a transformar o mundo em um lugar mais criativo, subversivo e sensível; acreditam que o ambiente de aprendizado pode ser leve e divertido; e que é preciso ter diversidade e multiplicidade na educação. Além disso, a metodologia que direciona todas as ações da empresa é baseada na ludificação e na gamificação.

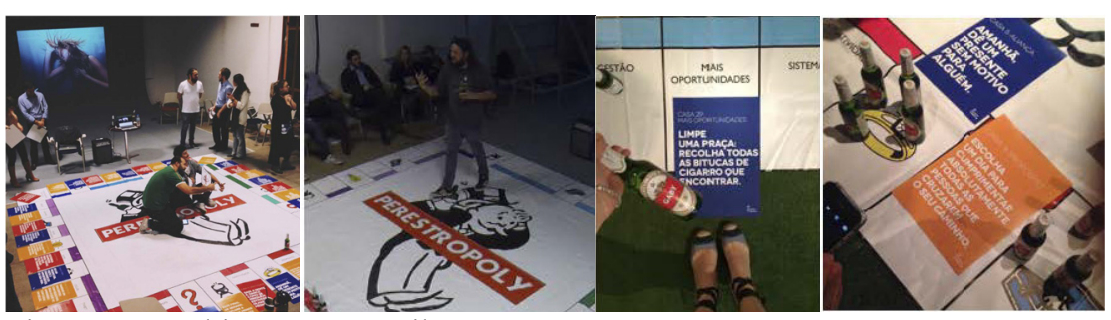

Figura 6 - Ambiente Perestroika.

Fonte: Perestroika ${ }^{2}$.

Já, a escola Quest to Learn (Figura 7), localizada em Nova York, tem como propósito promover a experiência pedagógica, usando a lógica dos jogos (SILVEIRA, 2016). Lemes e Sanches (2016) estudaram esse caso e mencionam que é a primeira escola do mundo a ter todo ensino baseado na gamificação. Os autores também relataram que os professores devem atuar como designer, orientador, pensador sistêmico, integrador do bem-estar, integrador de tecnologia e gestor. $\mathrm{O}$ aprendizado dos alunos é baseado nos valores da escola que são: aprendizado para o bem-estar e inteligência emocional; aprendizado para design e inovação; raciocínio sistêmico; aprendizado para pensamento crítico, julgamento e credibilidade; aprendizado utilizando metodologia de design; e aprendizado com tecnologia e ferramentas inteligentes. Além disso, dentre as estratégias chaves do aprendizado está inclusa a prototipagem. Logo, essa escola é um exemplo de caso que utiliza e relaciona as áreas estudadas nesta pesquisa: gestão de design, abordagem sistêmica, prototipagem de serviço e a gamificação como estratégia.

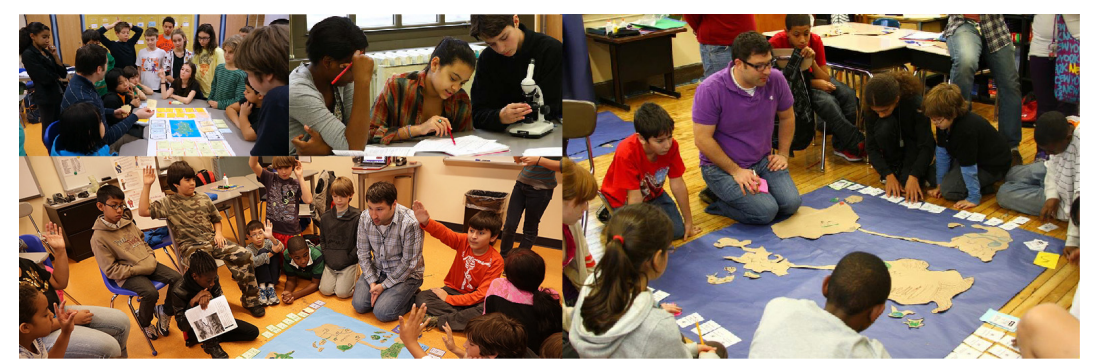

Figura 7 - Ambiente Quest to Learn.

Fonte: Quest to Learn ${ }^{3}$, Connected Learning ${ }^{4}$, Innove Edu (2018).

\footnotetext{
${ }^{2}$ Para mais informações: https://www.perestroika.com.br/peres-o-que/.

${ }^{3}$ Acesse: https://www.q21.org/.
} 
Cita-se, também, a escola Vittra Telefonplan School (Figura 8), localizada na Suécia, em que a proposta está direcionada aos espaços lúdicos, sem paredes, coloridos e com objetos abstratos na decoração. Por isso, no lugar das tradicionais mesas e carteiras, uma caverna para os momentos individuais de concentração, um laboratório para explorar cores, materiais e formas, e um palco para apresentar as descobertas. (SILVEIRA, 2016; BOSH; WENDT; GOMES, 2012).

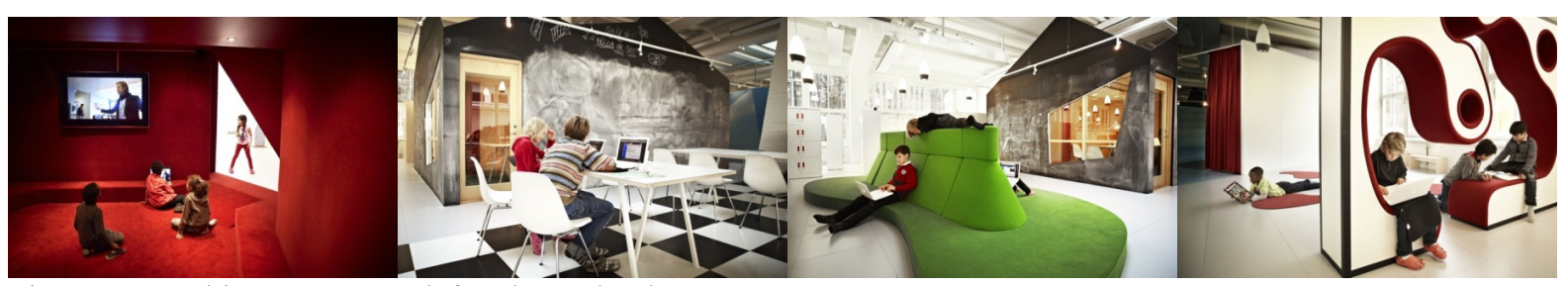

Figura 8 - Ambiente Vittra Telefonplan School.

Fonte: Vittra Telefonplan School ${ }^{5}$.

Assim, a Perestroika, a Quest to Learn e a Vittra Telefonplan School são exemplos de organizações educacionais que usam a gamificação ou a ludificação no nível estratégico da gestão de design, expandindo-as para os demais níveis tático e operacional. Schön (2000) aborda sobre o pensamento reflexivo e a importância desse para o ensino, em que é necessário as pessoas refletirem por meio da ação. Essa abordagem está relacionada com a metodologia dessas escolas citadas anteriormente, além disso, o designer também trabalha diretamente com o pensamento reflexivo. Assim, propõe-se a abordagem sistêmica na gestão de design, associada à prototipagem de serviço, para a visualização do processo de tomada de decisão para a aplicação de estratégias instrucionais em diferentes contextos. A seguir apresenta-se as principais considerações finais encontradas.

\section{Considerações finais}

O objetivo geral deste artigo, descrever as relações existentes entre a gestão de design sistêmica, a prototipagem de serviço e as estratégias instrucionais (ludificação e gamificação) direcionadas ao contexto de ensino-aprendizagem e/ou treinamentos em diferentes organizações, foi atingido por meio das três revisões tradicionais e sistemática da literatura realizadas. Essas revisões possibilitaram o mapeamento de estudos referentes à gestão de design, a abordagem sistêmica, ao processo de prototipagem de serviços e as estratégias instrucionais (ludificação e gamificação). Isso, por sua vez, proporcionou a compreensão das intersecções entre essas áreas contribuindo para identificar como ocorrem essas relações. $\mathrm{O}$ resultado desse entendimento está sintetizado na Figura 4 referente ao diagrama sistêmico que mostra as relações entre os conceitos. Além disso, a análise e o cruzamento dessas pesquisas foram relevantes para compreender que a gestão de design sistêmica associada a prototipagem de serviço auxiliou a entender como as estratégias instrucionais de ludificação e de gamificação estão inseridas nos níveis de gestão de design. Isso ficou ainda mais evidente ao analisar os três casos de organizações educacionais denominadas Perestroika, Quest to Learn e Vittra Telefonplan School. Esses casos permitem afirmar que a ludificação e a gamificação podem ser aplicadas em todos os níveis de gestão de design.

Nesta pesquisa, a gestão de design sistêmica (pela teoria e abordagem sistêmica) está associada a prototipagem de serviço e as estratégias instrucionais de ludificação e de gamificação (considerando o aspecto estratégico do design como fim para chegar às ideias e aos métodos que elevam a eficiência de gestão). Evidencia-se que a gestão de design sistêmica auxilia na prática de gestão, a identificar e colocar em prática a estratégia instrucional adequada para determinado contexto em organizações educacionais, sociais e corporativas. A prototipagem de serviço propicia o nível tático na visualização dos processos, seja no nível estratégico (para a equipe compreender a opinião dos demais e entender o que cada um está pensando por meio da representação gráfica, tornando as ideias, as opiniões e o processo mais tangíveis) ou no nível operacional (para a equipe compreender como está sendo a experiência dos usuários e identificar as falhas para aperfeiçoá-la). As estratégias instrucionais contribuem para a instrução, tanto da equipe quanto do usuário, e são compreendidas como estratégias

\footnotetext{
${ }^{4}$ Acesse: https://connectedlearning.uci.edu/wp-content/uploads/2018/01/Quest_to_Learn-sm.jpg.

${ }^{5}$ Para mais informações: https://www.archdaily.com/202358/vittra-telefonplan-rosan-bosch.
} 
de inovações tecnológicas para alcançar a inovação social, baseada na gestão de design, que pode impactar na estrutura das organizações em todos os níveis, mudando a forma como interagem, viabilizam e comportam novas relações, públicos, processos, práticas e outras formas de engajamento.

Por fim, evidencia-se como sugestão para futuras pesquisas a realização de revisões sistemáticas da literatura de 2019 até ao dia de hoje e principalmente na área de ludificação. Outra oportunidade encontrada é aplicar na prática a compreensão teórica aqui exposta em casos de organizações educacionais para dar continuidade nesta pesquisa, além de agregar conhecimento empírico aos casos já analisados neste artigo.

Systemic design management associated with service prototyping for decision making in the application of instructional strategies in different educational contexts: ludification and gamification

Abstract: This article aims to describe the relationships between systemic design management (SDM), service prototyping and instructional strategies (ludification and gamification) directed to the context of teaching-learning and/or training in different organizations. For this, three educational cases that apply these instructional strategies are mentioned. This research is theoretical in nature, qualitative approach, exploratory objectives. Data were collected through bibliographical research, including three literature reviews conducted from 2016 to 2018. The research found allowed us to understand the relationships between these areas, resulting in a systemic diagram. SDM assists in the practice of management, to identify and put into practice the appropriate instructional strategy for a given context. Service prototyping provides the tactical level in the visualization of processes, whether at the strategic or operational level. Instructional strategies contribute to team and user instruction, and can be applied at all SDM levels.

Keywords: Systemic design management; service prototyping; instructional strategies; ludification; gamification

\section{Referências bibliográficas}

ALVES, Flora. Gamification: como criar experiências de aprendizagem engajadoras. 2. ed. São Paulo: DVS Editora, 2015.

AROS, Kammiri Corinaldesi; FIGUEIREDO, Luiz Fernando Gonçalves de. Gestão de design para inovação social: uma possível relação. In: INTERNATIONAL CONFERENCE ON INTEGRATION OF DESIGN, ENGINEERING AND MANAGEMENT FOR INNOVATION, IDEMI, 4., 2015, Florianópolis. Anais eletrônicos [...]. Florianópolis: UDESC, 2015. Disponível em: http://janainaramos.com.br/idemi2015/anais/05/141070.pdf. Acesso em: 28 jul. 2020.

BERTALANFFY, Ludwig Von. Teoria geral dos sistemas: fundamentos, desenvolvimento e aplicações. 8. ed. Petrópolis: Vozes, 2014.

BEST, Kathrin. Fundamentos de gestão do design. Porto Alegre: Bookman, 2012.

BLOMKVIST, J. Conceptualising Prototypes in Service Design. Trabalho de Conclusão de Curso (Graduação de Licenciatura em Filosofia) - Department of Computer and Information Science, Faculty of Arts and Sciences, Linköping University, Suécia, 2011.

BOHREN, Meghan A. et al. Formative research and development of innovative tools for "Better Outcomes in Labour Difficulty" (BOLD): study protocol. Reproductive Health, v. 12, 2015.

BONSIEPE, Gui. Design, Cultura e Sociedade. São Paulo: Blucher, 2011.

BOSH, Rosan; WENDT, Kim; GOME, Patrícia. Escolas suecas aproximam pedagogia e design. In: PORVIR. Inovações em Educação. São Paulo: Porvir, 2012. Disponível em: http://porvir.org/escolasueca-aproxima-pedagogia-design/. Acesso em: 28 jul. 2020. 
BROWN, Tim. Design thinking: uma metodologia ponderosa para decretar o fim das velhas ideias. Rio de Janeiro: Elsevier, 2010.

CAPRA, Fritjof. A teia da vida: uma nova compreensão científica dos sistemas vivos. 12. ed. São Paulo: Cultrix, 2010.

CARLAN SÁ, Neusa Maria. 0 lúdico na ciranda da vida adulta. 2004. Dissertação (Mestrado em Educação) - Programa de Pós-graduação em Educação, Universidade do Vale do Rio dos Sinos, São Leopoldo, 2004.

CASTRO, Gabriela Varanda de. Jardins comunitários de Nova York: um método para recomendações baseado no design de experiência. 2014. Tese (Doutorado em Design) -

Departamento de Artes e Design, Pontifícia Universidade Católica do Rio de Janeiro, Rio de Janeiro, 2014.

COHEN, Aaron M. The gamification of education. Futurist, v. 45, n. 5, p. 16-17, 2011.

CONFORTO, Edivandro Carlos; AMARAL, Daniel Capaldo; SILVA, Sérgio Luis da. Roteiro para revisão bibliográfica sistemática: aplicação no desenvolvimento de produtos e gerenciamento de projetos. In: CONGRESSO BRASILEIRO DE GESTÃO E DESENVOLVIMENTO DE PRODUTO, 8., 2011, Porto Alegre.

Anais [...]. Porto Alegre: UFRGS, 2011.

DALLAGNOL, Vinícius. A inserção da gamificação no processo de gestão de design. 2016. Dissertação (Mestrado em Design) - Centro de Comunicação e Expressão, Programa de PósGraduação em Design, Universidade Federal de Santa Catarina, Florianópolis, 2016.

Lúdico, Ludismo, Ludificação, Ludicidade, Ludificar, Joguificar, Joguificação, Gamificar, Gamificação. In: DICIONÁRIO Priberam da Língua Portuguesa, DPLP. Porto: Lello Editores, 2013. Disponível em: https://dicionario.priberam.org/. Acesso em: 28 jul. 2020.

DUTRA, Jussara. A inovação empresarial depende do engajamento das pessoas. In: SENIOR. Notícias. [S. I.]: Senior Sistemas, 2013. Disponível em: https://www.senior.com.br/noticias/ainovacao-empresarial-depende-do-engajamento-das-pessoas. Acesso em: 26 ago. 2017.

FERREIRA, Caroline Nascimento; SANTOS, Gabriela Damázio dos. Design Lúdico: definições de uma estrutura interativa. In: CONGRESSO BRASILEIRO DE PESQUISA E DESENVOLVIMENTO EM DESIGN, 11., 2014, Gramado. Anais eletrônicos [...]. São Paulo: Blucher, 2014, p.1-9. Disponível em: http://www.ufrgs.br/ped2014/trabalhos/trabalhos/833_arq2.pdf. Acesso em: 28 jul. 2020.

FILATRO, Andrea. Design instrucional na prática. São Paulo: Pearson Education do Brasil, 2008.

FIGUEIREDO, Luiz Fernando Gonçalves de; FERREIRA, Alais Souza; CONTI, Paolo. Prototipagem de Serviço relacionada à Gamificação e Gestão de Design por uma Abordagem Sistêmica: análise bibliométrica. In: CONGRESSO DE PESQUISA \& DESENVOLVIMENTO EM DESIGN, 13., 2018, Joinville. Anais [...]. São Paulo: Blucher, 2018, v. 1. p. 2610-2624.

FERREIRA, Alais Souza; FIGUEIREDO, Luiz Fernando Gonçalves de; OURIVES, Eliete Auxiliadora Assunção. Abordagem Sistêmica associada à gamificação como uma ferramenta de prototipagem de serviços. In: COLÓQUIO INTERNACIONAL DE DESIGN, 4., 2017, Belo Horizonte. Anais eletrônicos [...]. São Paulo: Blucher, 2018. Disponível em: http://www.proceedings.blucher.com.br/articledetails/28115. Acesso em: 28 jul. 2020.

FORMANSKI, Francieli Naspolini. Aplicabilidade da gamificação no contexto empresarial. 2016. Dissertação (Mestrado em Engenharia e Gestão do Conhecimento) - Centro Tecnológico, Programa de Pós-Graduação em Engenharia e Gestão do Conhecimento, Universidade Federal de Santa Catarina, Florianópolis, 2016.

GIL, Antônio Carlos. Métodos e Técnicas de Pesquisa Social. 5. ed. São Paulo: Atlas, 2014.

HUIZINGA, Johan. Homo ludens: o jogo como elemento da cultura. 4. ed. São Paulo: Perspectiva, 2014.

HOSS, MAURICIO JUNIOR. Prototipagem de serviços: um estudo exploratório com foco na iluminação de habitações de interesse social. 2014. Dissertação (Mestrado em Design) - Setor de Artes, Comunicação e Design, Universidade Federal do Paraná, Curitiba, 2014. 
INNOVE EDU. Quest to Learn: cidade de Nova York e Institute of Play. In: INNOVE EDU. São Paulo: Gita Tecnologia, 2018. Disponível em: http://www.innoveedu.org/pt/quest-to-learn. Acesso em: 28 jul. 2020.

JENKINS, Henry; CLINTON, Katie; PURUSHOTMA, Ravi; ROBISON, Alice J.; WEIGEL, Margaret. Confronting the challenges of participatory culture: media education for the 21th century. Chicago: MacArthur Foundation, 2006. Disponível em: https://www.macfound.org/media/article_pdfs/JENKINS_WHITE_PAPER.PDF. Acessado em: 28 jul. 2020.

KRUCKEN, Lia. Design e território: valorização de identidades e produtos locais. 1. ed. São Paulo: Nobel, 2009.

KOTLER, Philip. Administração de Marketing. São Paulo: Prentice Hall, 2005.

LAURO, Aline Bertolini. Abordagem sistêmica e gestão de design na formação de uma rede integradora de serviços. 2014. Dissertação (Mestrado em Design) - Centro de Comunicação e Expressão, Programa de Pós-Graduação em Design e Expressão Gráfica, Universidade Federal de Santa Catarina, Florianópolis, 2014.

LEMES, David de Oliveira; SANCHES, Murilo Henrique Barbosa. Gamificação e Educação: Estudo de caso da Escola Quest to Learn. In: SIMPÓSIO BRASILEIRO DE JOGOS E ENTRETENIMENTO DIGITAL, 15., 2016, São Paulo. Proceedings eletrônicos [...]. São Paulo: SBC, 2016. Disponível em: http://www.sbgames.org/sbgames2016/downloads/anais/157723.pdf. Acesso em: 28 jul. 2020.

MORIN, Edgar. 0 método 1: a natureza da natureza. Porto Alegre: Sulina, 2005.

MORITZ, Stefan. Service Design: practical access to an evolving fiel. [S. I.]: Köln International School of Design, 2005.

MOZOTA, Brigitte Borja de. Gestão do design: usando o design para construir o valor de marca e inovação corporativa. Porto Alegre: Bookman, 2011.

NAVARRO, Gabrielle. Gamificação: a transformação do conceito do termo jogo no contexto da pósmodernidade. 2013. Dissertação (Mestrado em Comunicação) - Universidade Anhembi Morumbi, São Paulo, 2016.

NEGRINE, Airton. O lúdico no contexto da vida humana: da primeira infância à terceira idade. In: Brinquedoteca: a criança, o adulto e o lúdico. 1. ed. Petrópolis: Vozes, 2000.

PINK, Dan. The puzzle of motivation. In: TED GLOBAL CONFERENCE. [S. I.]: TED Conferences, 2009. Disponível em: http://www.ted.com/talks/dan_pink_on_motivation\#t-759582. Acesso em: 28 jul. 2020.

RAMOS, Cosete. Construção de materiais de ensino-aprendizagem: uma abordagem sistêmica. Brasília: Ministério da Educação e Cultura, Departamento de Ensino Fundamental, Departamento de Documentação e Divulgação, 1978.

RAU, Christiane; ZBIEK, Anna; JONAS, Julia M. Creating Competitive Advantage from Services: a Design Thinking Case Study from the Commodities Industry. Research Technology Management, Arlington, v. 60, n. 3, p. 48-56, May. 2017.

RIBEIRO, Débora. Lúdico. In: DICIONÁRIO Online de Português, DICIO. Porto: 7Graus, 2018. Disponível em: https://www.dicio.com.br/ludico/. Acesso em: 28 jul. 2020.

ROLOFF, Eleana Margarete. A importância do lúdico em sala de aula. In: X SEMANA DE LETRAS, 70, 2010, Porto Alegre. Anais eletrônicos [...]. Porto Alegre: PUC-RS, 2010. Disponível em:

http://ebooks.pucrs.br/edipucrs/anais/Xsemanadeletras/comunicacoes/Eleana-Margarete-Roloff.pdf. Acesso em: 28 jul. 2020.

SCHÖN, Donald A. Educando o Profissional Reflexivo: um novo design para o ensino e a aprendizagem. Porto Alegre: Artmed, 2000.

SIGNIFICADOS. Lúdico. In: SIGNIFICADOS. Porto: 7Graus, 2014. Disponível em: https://www.significados.com.br/ludico/. Acesso em: 28 jul. 2020. 
SILVA, Carina Scandolara da; FIGUEIREDO, Luiz Fernando Gonçalves de. Abordagem sistêmica da gestão de design: conceituação e aplicação. In: CONFERÊNCIA INTERNACIONAL DE DESIGN, ENGENHARIA E GESTÃO PARA INOVAÇẪO, 4., 2015, Florianópolis. Anais eletrônicos [...]. Florianópolis: UDESC, 2015, p. 1063-1074. Disponível em:

http://janainaramos.com.br/idemi2015/anais/04/143419.pdf. Acesso em: 31 ago. 2018.

SILVEIRA, Fabio. Design \& educação: novas abordagens. In: MEGIDO, V. F. A revolução do design: conexões para o século XXI. São Paulo: Editora Gente, 2016. p.116-131.

VIANNA, Ysmar; VIANNA, Maurício; MEDINA, Bruno; TANAKA, Samara. Gamification, inc: como reinventar empresas a partir de jogos. 1 ed. Rio de Janeiro: MJV Press, 2013.

ZIMMER SANTOS, Grazielli Faria; HOFFMANN, Micheline Gaia. Pursuing effectiveness in public administration: a proposal for a method to design and implement public services in the city of florianopolis. NAVUS-Revista de Gestão e Tecnologia, v. 6, n. 1, p. 88-105, 2016. 